\title{
Identificación y efectos de los diferentes métodos del amamantamiento restringido sobre la funcionalidad ovárica posparto en hembras bovinas mestizas doble propósito
}

\section{Identification and effects of different methods of restricted suckling on postpartum ovarian function in female cattle crossbred dual purpose}

\author{
Vargas Ángel Gino Odair ${ }^{1}$ y Jaramillo Hernández Dumar Alexander ${ }^{2}$ \\ ${ }^{1}$ Medico Veterinario Zootecnista Esp. \\ ${ }^{2}$ Medico Veterinario Zootecnista, Dipl. Esp., (c)MSc. Docente Universidad de los \\ Llanos. Grupo de Investigación en Farmacología Experimental y Medicina Interna \\ - ÉLITE \\ dumar.jaramillo@unillanos.edu.co
}

Recibido 05 de Marzo 2013, Aceptado 02 Abril 2013

\section{RESUMEN}

El anestro posparto es una de las causas principales que limitan la eficiencia productiva y reproductiva de las ganaderías doble propósito en las regiones tropicales donde se utiliza el ternero como apoyo somatosensorial para la producción de leche. El amamantamiento continuo y la presencia constante del ternero inhiben la secreción de $\mathrm{GnRH}$ en el Hipotálamo debido al incremento de los opioides endógenos y del estradiol en hipotálamo e hipófisis, afectando negativamente la especificidad de la $\mathrm{GnRH}$. El amamantamiento restringido es utilizado como una opción de manejo para reducir los efectos negativos mamogénicos del ternero sobre la actividad ovárica posparto. Los diferentes modelos de amamantamiento restringido (destete precoz, destete temporal, amamantamiento una vez por día, enlatado) utilizados en ganaderías Doble propósito se diferencian en la técnica y procedimiento, obteniendo en muchos casos diferentes resultados.

Palabras clave: Amamantamiento restringido, efecto mamogénico, ovarios, ganadería doble propósito. 


\begin{abstract}
In tropical regions, postpartum anestrus is one of the main causes that limit production and reproductive efficiency of the double purpose cattle raising where the calf is used as somatosensory support for milk production. The continuous breastfeeding and the constant presence of the calf inhibit the secretion of $\mathrm{GnRH}$ in the hypothalamus due to increased endogenous opioids and estradiol in the hypothalamus and pituitary, negatively affecting the specificity of $\mathrm{GnRH}$. The restricted breastfeeding is used as a management option to reduce the negative suck originated effects of the calf on postpartum ovarian activity. Different models of restricted feeding (early weaning, weaning time, feeding once per day, canned) used in double purpose cattle rising is different in technique and procedure, often obtain different results.
\end{abstract}

Keywords: Restricted suckling, suck originated effect, ovaries, dual-purpose cattle.

\title{
INTRODUCCIÓN
}

La ganadería doble propósito en el departamento del Meta se encuentra ubicada en un $90 \%$ en el piedemonte llanero, con alto índice de mestizaje en los animales que la integran. La actividad ganadera (carne y leche) representó, para el año 2008 , el $25 \%$ del valor de la producción agropecuaria nacional y el $62 \%$ del sector pecuario; siendo así, el sistema doble propósito el constituyente más dinámico de la ganadería colombiana, aportando el 57\% de la producción nacional de leche; y en el piedemonte llanero el $90 \%$ de la producción de leche (Secretaría de Agricultura y Ganadería, Cadenas Productivas del Meta, 2008).

En el departamento del Meta la población de hembras mayores de tres años, en el 2007, fue de 474.248, el 58\% de estas hembras están en ordeño, las cuales producen 962.274 litros de leche día, con una producción promedio de 3,5 litros de leche vaca día; pero esa producción no es la misma que se comercializa, porque los terneros, que en el 2007 ascendieron a 274.010 menores de un año, se toman 749.244 litros de leche/día en el sistema de explotación cría y doble propósito. 
Con base en esta inferencia, la leche que se comercializa equivale a 213.030 litros día en el departamento del Meta (Secretaría de Agricultura y Ganadería del Meta, Evaluaciones agropecuarias, 2007).

La nutrición y lactancia son dos factores importantes que controlan la duración del período de anestro posparto (Randel, 1990; Williams, 1990); como acción estratégica la nutrición y la lactancia deben mejorar el desempeño reproductivo de las vacas, buscando un equilibrio entre producción láctea, condición corporal de las vacas durante la lactancia y alcanzar un buen peso al destete de terneros; es allí, donde el punto de equilibrio entre estos dos factores debe permitir el rendimiento necesario para mantener una ganadería productiva, si alguno de estos puntos pierde su nivel óptimo de eficiencia, el sistema doble propósito perdería su productividad. Esto indudablemente involucra el efecto mamogénico del ternero sobre su madre y el amamantamiento restringido como alternativa del manejo del ternero, para lograr el equilibrio combinado entre ganancia peso ternero-madre, ternero año y producción láctea (Preston y Vaccaro, 1989). En este orden de ideas, este trabajo realiza un proceso critico reflexivo sobre la estrategia del amamantamiento restringido como alternativa de manejo de hatos doble propósito y su impacto sobre el desempeño reproductivo de las vacas en periodo posparto; mostrando la posibilidad de implantación de actividades que mejoren los rendimientos productivos y reproductivos del sistema de producción doble propósito bovino en Colombia (Galeano, 2010).

\section{EFECTO DEL AMAMANTAMIENTO RESTRINGIDO SOBRE EL DESEMPEÑO REPRODUCTIVO POSPARTO EN LAS HEMBRAS BOVINAS MESTIZAS DOBLE PROPÓSITO}

\section{Etología, relación vaca-ternero conducta del animal}

La conducta animal es una consecuencia genético-ambiental, por ello se le estudia desde los puntos de vista evolutivos y adaptativo; por otra parte, la etología es una ciencia que estudia la conducta animal, sus fundamentos genéticos y neuroendocrinos; aunque como ciencia, es un campo relativamente nuevo. Hoy en 
día se aceptan los términos: psicología humana y psicología animal, como ramas de la etología, incluso habiéndose derivado de ésta otras ramas como la ecología de la conducta (behavioral ecology) y el bienestar animal (animal welfare); en este orden de ideas, el bienestar animal y las buenas prácticas ganaderas se enfocan sobre la solución a problemas causados al animal doméstico por el abuso del hombre (Landaeta, 2011).

Los sistemas de producción animal intensivos, desarrollados por los países con alta tecnología, han conllevado al confinamiento y prácticas de manejo inadecuado, que se traducen en daños a la integridad física y psicológica del animal doméstico. Estos daños se expresan en disminuciones en la productividad, representados en términos de calidad y cantidad, incluso llegando a estar comprometida la salud pública. Para el caso de los países subdesarrollados y tropicales, con predominio del pastoreo, presencia de sangre cebú, mestizaje indiscriminado, prácticas de manejo rudimentarias y bajo nivel cultural; siendo esta última característica, la causa que promueve la permanencia y el afloramiento de conductas ancestrales, las cuales influyen en la toma de decisiones en los sistemas de producción animal. Es por ello, que el estudio de la etología y bienestar animal viene siendo el pilar de desarrollo y evolución del manejo animal en los sistemas de producción; introduciéndose hoy día, esta ciencia en la formación académica de los profesionales del campo (Landaeta, 2011).

La organización social del rebaño puede afectar aspectos reproductivos como: el retorno a la ciclicidad, la expresión de celo y la eficiencia reproductiva. El retorno a la ciclicidad es afectado directamente, puesto que el acceso limitado a alimentos (especialmente en las primíparas) y la consecuente pérdida de peso conlleva a prolongar el intervalo parto-celo. En cuanto a la expresión del celo, las vacas cebuínas dominantes tienden a expresar menos el celo que las subordinadas; ello ocurre, porque aún estando en celo, las vacas dominantes tardan en dejarse montar pues no permiten que vacas de rangos sociales inferiores las monten. Por el contrario, en estudios con ganado Angus, las vacas dominantes en presencia o ausencia del toro son montadas en las primeras tres a seis horas de iniciado el 
celo, observando una menor influencia de segregación entre las hembras del hato bovino estudiado (Landaeta, 2011). Aún este tipo de estudios no han sido desarrollados en zonas tropicales. Tanto en hembras de razas Bos taurus como Bos indicus, la expresión del celo parece variar según la presencia o ausencia del toro; cuando el toro está ausente, las vacas se montan más y por mayor tiempo, que cuando el toro está presente. En este sentido, el uso de toros calentadores resulta de mayor ayuda como efecto bioestimulante, que como detector de celo (Landaeta, 2011).

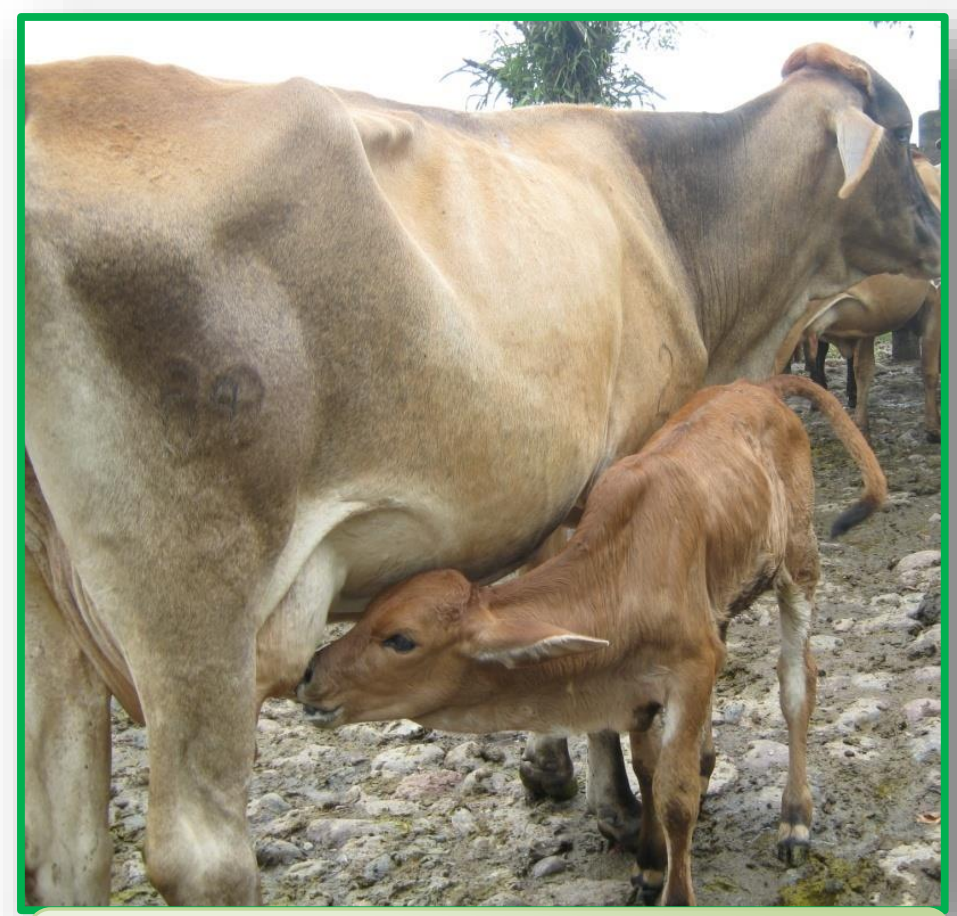

Fotografía 1. Madre mestiza amantando su ternero después del ordeño en el trópico Fotografía: María Ligia Roa Vega, (2011)
La conducta materna se define como la suma de todos los patrones de comportamiento que la madre exhibe en función de asegurar la sobrevivencia de la cría, por lo que se extiende desde el parto hasta el destete de la cría; pero es un estado que varía entre especies, razas e individuos. En términos zootécnicos, la conducta materna tiene repercusiones en la lactancia (en vacas de genotipos tropicales) y en la eficiencia reproductiva.

El amamantamiento es el comportamiento del ternero para propiciar la obtención de leche de una mamila o teta; esta conducta incluye el localizar y reconocer a un potencial proveedor de leche (generalmente la madre). Los primeros movimientos del ternero luego de haber nacido, por lo general consisten en dirigirse hacia la madre y localizar las tetas, en ocasiones con movimientos aparentemente aleatorios de su hocico (Hafez, 1996); por otra parte, en el ganado productor de carne, los terneros recién nacidos inician su amamantamiento aproximadamente 90 minutos después de nacer (Lidfors y Jensen, 1994). 
En particular con las razas cebuínas, la falta de conducta materna puede conllevar a la muerte del becerro y en consecuencia al cese de la lactancia, lo cual es un problema. La inducción de la habilidad materna ha sido reportada en ovejas mediante el uso combinado de hormonas (estradiol y oxitocina), elementos sensoriales de la conducta (visión, olfato) y manipulación de genitales, tales resultados no han podido ser replicados en vacunos (Landaeta, 2011). En cuanto a la eficiencia reproductiva, la sensibilidad hipotalámica de la vaca posparto y en consecuencia, la reactivación ovárica posparto puede ser influenciada por el amamantamiento; el establecimiento del vínculo vaca-ternero con una representación de comportamiento estrecho, de interacción fuerte, tiende a generar problemas como el anestro, el cual se complica en ausencia del toro. Los órganos de los sentidos como la visión y el olfato son fundamentales para el reconocimiento del ternero por parte de su madre dando origen a la interacción del vínculo vaca-ternero conllevando a la inhibición de la hormona luteinizante (LH) (Landaeta, 2011).

Fundamentalmente, existen dos maneras de prevenir el efecto negativo del vínculo vaca-ternero: A) mediante el uso intenso de la bioestimulación (efecto de la presencia del macho), y B) mediante el manejo de la vaca y el ternero con el fin de reducir al mínimo el contacto (vista y olfato) de la vaca con el ternero. En el primer caso, se busca que en presencia del reproductor o de toros calentadores, se estimule a la reanudación de la ciclicidad posparto hasta 30-45 días posparto. En el segundo caso, es determinante que la vaca no observe ni huela a su becerro, tan solo en el momento de apoyo para la estimulación de descarga de oxitocina, a la hora del ordeño. Una vez instaurado el anestro, la separación temporal del ternero (48-72 horas) es una estrategia para lograr el retorno a la ciclicidad (Landaeta, 2011).

\section{Fisiología de la hembra bovina posparto}

La hembra bovina debe tener un intervalo entre partos de 12 a 13 meses, destetar un becerro por vaca cada año y producir una cantidad adecuada de leche para su venta. Para lograr el primer objetivo, las vacas deben restablecer su actividad 
ovárica cíclica entre 65 y 85 días después del parto, con la finalidad de tener al menos dos oportunidades de servirlas y la concepción se presente entre los días 85 y 115 posparto. Obtener un parto por vaca cada año en los hatos doble propósito, es difícil de lograr con las condiciones actuales de manejo extensivo que se desarrollan en la región Orinoquia, esta condición es causada por prolongados períodos de anestro posparto; afectando la eficiencia reproductiva y productiva de las ganaderías doble propósito de la región tropical (Pérez et al., 2001). Esto se refleja en tasas anuales de parición de 45 a 55\% (Román, 1995) e intervalos entre partos promedio mayores a 540 días (Villa y Arreguín, 1993). La duración del anestro postparto en tales condiciones se prolonga hasta 150-250 días (Santos et al., 1995; Ruiz y Olivera, 1999; Villagómez et al., 1999) y es el principal factor que afecta el intervalo entre partos (Williams et al., 1996; Ruiz y Olivera, 1999).

Uno de los diferentes períodos de mayor importancia en el ciclo reproductivo, considerado el factor más limitante en la eficiencia reproductiva, es el periodo comprendido entre el parto y la concepción o días abiertos (Salgado et al., 2007).

El puerperio es un proceso fisiológico de modificaciones que ocurren en: útero, cérvix, vagina, vulva y ovarios en la fase inmediata después del parto, cuando estos órganos se recuperan de las transformaciones sufridas durante la gestación y el parto deben prepararse para una nueva gestación. Malven (1984) limitó la finalización del puerperio al primer estro posparto en el que se puede restablecer la gestación; para ello la involución anatómica e histológica uterina debe haber completado y además el eje hipotálamo-hipófisis-gonadal debe funcionar normalmente para permitir el estro, la ovulación, la concepción, la implantación embrionaria, la formación y persistencia del cuerpo lúteo de gestación y la preñez a término.

\section{Puerperio propiamente dicho}

Está caracterizado por la regresión del útero a su condición normal pregestacional, esto ocurre por eliminación, disolución y reabsorción decidual que determina 
evidentemente disminución del volumen del órgano; según Villagómez et al., (1999) se divide en tres subfases:

a. Puerperio temprano: desde la eliminación de las secundinas hasta el día noveno, la regresión uterina está concluida, las barreras defensivas se han completado.

b. Puerperio clínico: hasta el día 21 por parto, el útero involuciona hasta aproximadamente el tamaño del órgano no grávido.

c. Puerperio total: aproximadamente seis semanas posparto, donde las modificaciones del endometrio causadas por la gestación ya no existen, se ha concluido la regeneración histológica completa.

\section{Modificaciones que suceden durante el puerperio en la vaca}

El aparato reproductor de la hembra sufre modificaciones circunstanciales luego del parto, siendo sometido a cambios fisiológicos que buscan un restablecimiento para su normal funcionamiento y acondicionamiento de una nueva gestación (Corbellini et al., 2011).

Restablecimiento de la forma del cérvix. El cérvix es una barrera importante contra la invasión de bacterias desde el exterior hacia la cavidad uterina; por lo tanto, es obvio que el cierre del canal cervical es necesario y la recuperación de su estructura después del parto debe ser rápida para que en breve tiempo se instaure una nueva gestación (Perea et al., 1997). Hay solo un estudio que midió el cierre cervical describiendo el proceso durante los primeros 10 días del posparto y se observó que la disminución del diámetro cervical iba de 25 a 5,2 cm, esta disminución se produce en los primeros dos días (Wehreden et al., 2003).

En un estudio de Van Engelen et al., (2009) en el cual se midió el cérvix, en vacas inducidas al parto y/o aborto, encontrando a partir de la primera hora de expulsado el feto y hasta los tres días posparto el cérvix tenía un diámetro de $6.2( \pm 0.7) \mathrm{cm}$; a las 14.8 horas posparto el diámetro era de $9.0( \pm 1.0) \mathrm{cm}$; a las 33.2 horas posparto el diámetro era de $5.3( \pm 1.0) \mathrm{cm}$ y a las 48 horas posparto era de 2.0 a $9.0 \mathrm{~cm}$. A 
partir de la expulsión del feto y las membranas fetales, la involución cervical se puede separar en varias etapas (Hafez, 2000):

a. Entre las 4 y 16 horas posparto se produce una reducción progresiva de la luz cervical.

b. Al segundo día posparto se produce una lenta reducción sin una reconstrucción de los anillos y sus pliegues, pero se nota a la palpación vaginal que está formado con una pequeña elevación anular, permitiendo el pasaje de uno a dos dedos. A la palpación rectal es una estructura que comienza a hacerse firme (Hafez, 2000).

c. Alrededor del séptimo día posparto se ha mantenido su lenta reducción con una formación de los anillos y pliegues que son más evidentes, difícilmente es franqueable por un dedo.

d. Entre el día 10 al 14 posparto presenta una discreta reapertura, estando ésta en relación directa con la primera ovulación posparto con gran presencia de granulocitos y eosinófilos en la túnica mucosa; las estructuras anulares y los pliegues ya están formados. Ya se encuentra en cavidad pelviana, dependiendo a veces de la cantidad de partos y del tamaño de la hembra bovina (Hafez, 2000).

e. Alrededor de los días 25 a 30 posparto alcanza ya su estado pregestacional; con todas las formaciones anulares completas y con una ubicación pélvica (Hafez, 2000).

Involución uterina. La involución uterina es la recuperación del órgano de un estado pregestacional, luego de los cambios que presenta durante el período gestacional y el parto; se puede describir como un regreso a la normalidad en cuanto a su tamaño, consistencia, tono y ubicación. Entre los días 15 y 17 posterior al parto, el tamaño del útero es ligeramente superior al del útero no preñado. En cada ciclo reproductivo (después de cada parto) el útero aumenta de tamaño debido a los cambios que sufre durante la preñez, pero nunca alcanza de manera total a su estado pre gravídico; este aumento siempre guarda una relación de 1:1,2 (Domínguez et al., 2008). 
El cuerno uterino no gestante regresa a su tamaño pregestacional casi completamente mientras el cuerno que llevó adelante la gestación, así como el cérvix, permanecen ligeramente más grandes que antes de la gestación (Domínguez et al., 2008).

Involución caruncular y reparación endometrial. Durante el parto las continuas variaciones de la presión intrauterina causadas por las contracciones actúan sobre los placentomas, ejerciendo fuerzas de presión y descompresión, despegando el epitelio de las vellosidades de las criptas maternas ramificadas (Sumano et al., 1996). Durante la fase expulsiva proximal a los pedúnculos de las carúnculas se producen los primeros fenómenos de desprendimiento. Estos son debidos a la presión ejercida por los placentomas contra el feto y las variadas contracciones (Cavestany, 1985).

Ciclo de eliminación de los loquios. Los loquios están formados principalmente por acumulación de fluidos placentarios, sangre, restos tisulares y exudación endometrial. La sangre proviene de hemorragias capilares en el sitio donde se necrosan las carúnculas (Gaona et al., 2008). Las contracciones uterinas son más frecuentes que intensas y participan activamente al vaciamiento del útero. Un cierre parcial del cérvix sucede en este período y se produce el día 10 del posparto. A partir del día 10 hasta el 15 posparto la involución y el tono uterino aumentan y coincide con la primera onda folicular, que favorece la expulsión de restos de loquios a través del cuello uterino (Gaona et al., 2008). Después del día 18 a 20 posparto, las descargas uterinas son raras. (Tennant et al., 1967) observó que 30 a $35 \%$ de las vacas presentan una descarga vulvar de 50 a $200 \mathrm{ml}$ entre el día 10 y 20 posparto, contra sólo 2 a 5\% entre el día 30 a 50 posparto; en estas últimas eso es probablemente debido a la persistencia de una infección uterina.

Reinicio de la ciclicidad ovárica después del parto. La gestación se caracteriza por concentraciones elevadas de progesterona; en la fase final de la misma, hay una gran producción de estrógenos placentarios que inhiben a nivel hipotalámico el ritmo secretorio pulsátil de $\mathrm{GnRH}$, como consecuencia ante la ausencia de pulsos de $\mathrm{GnRH}$ se produce una rápida disminución de la cantidad y contenido de 
mRNA necesario para la síntesis de las subunidades $\alpha$ y $\beta$ de la hormona $\mathrm{LH}$, con una disminución gradual de la misma a nivel hipofisiario; se estima que durante la gestación las reservas hormonales de LH disminuyen hasta un 95\% (Montañés et al., 2005).

Normalmente pocos días después del parto tiene lugar la liberación de la hormona folículo estimulante (FSH) por parte de la hipófisis anterior, lo que conduce al posterior reinicio de la función cíclica ovárica (Bertha, 2002). Lamentablemente aquellos folículos dominantes (FD) que son los que logran un mayor desarrollo y que se jerarquizan en las primeras de las dos o tres ondas foliculares ováricas fallan en alcanzar el tamaño ovulatorio debido a insuficientes niveles de secreción pulsátil de la hormona luteotrópica (LH) secretada por la misma glándula hipofisiaria. Por el contrario, la FSH presente en la circulación desde los primeros días después del parto (dos a tres días), alrededor del quinto día posparto sus niveles plasmáticos se elevan y se evidencian fluctuaciones (Ptazynaska, 2007). Estas descargas de FSH, en los primeros días son suficientes para producir el reclutamiento, selección y dominancia de los folículos antrales, permitiendo al mismo tiempo la formación de vasos en las tecas, creando nuevos receptores para FSH en la granulosa, Interviniendo en el mecanismo de la esteroideogénesis (Gigli et al., 2006).

Debido a los estrógenos producidos por las células de la granulosa de los folículos, se estimula la adenohipófisis para producir más LH, hasta obtener un pico ovulatorio de gran amplitud pero de duración breve, pero que induce a la ovulación. El primer folículo dominante en el posparto en general aparece en el ovario contralateral al correspondiente al cuerno uterino pregrávido. Posiblemente debido a un efecto inhibitorio local por la regresión del cuerpo lúteo de gestación en el ovario ipsilateral al cuerno uterino gestante. Los primeros ciclos son cortos (8-15 días), caracterizados por una fase luteínica irregular, la regresión precoz del cuerpo lúteo aparentemente se debe a la liberación prematura de PGF $2 \alpha$, que a su vez es el resultado de la falta anterior de progesterona entre el parto y la primera ovulación (Gigli et al., 2006). 


\section{Efecto mamogénico sobre la función ovárica}

El amamantamiento juega un papel importante en gobernar los ciclos reproductivos de las hembras y su efecto indeseable debe ser debido a factores directos como el estímulo de la glándula mamaria y la liberación de algunas hormonas o inhibición de la liberación de otras, lo que interfiere con el reinicio de la actividad ovárica (Sergio, 2006). El amamantamiento puede atrasar el inicio de la actividad ovárica o la presentación del primer estro posparto en vacas de leche y de carne, de manera independiente o interactuando con otros factores (Acosta et al., 1983; García et al., 1984; Hinshewood et al., 1985; Henao et al., 2000).

La primera ovulación posparto de la mayoría de las vacas productoras de carne que amamantan a su becerro no se acompaña de conducta de estro (Werth et al., 1996) y frecuentemente es seguido por un cuerpo lúteo de vida media corta (Werth et al., 1996; Yavas et al., 1999). Se ha reportado que los cuerpos lúteos de vida media corta se presentan en la mayoría de las vacas productoras de carne (66-100\%) (Stagg et al., 1995), de manera independiente de la duración del anestro (Mukasa et al., 1991); y se caracterizan porque el cuerpo lúteo que se forma es pequeño, secreta menor cantidad de progesterona (Yavas et al., 1999), responde en menor grado a las gonadotropinas y sólo presentan una oleada folicular (Stagg et al., 1995; Yavas et al., 2000). Estos ciclos estrales cortos, son una de las principales causas de la baja tasa de concepción a primer servicio en vacas con baja condición corporal (Stagg et al., 1995).

La duración del anestro postparto es una causa principal que afecta la eficiencia reproductiva y productiva de las explotaciones bovinas de doble propósito en las regiones tropicales. Su duración se incrementa por efecto del amamantamiento y la presencia continua del becerro, al inhibir la secreción de GnRH y LH. Existen evidencias que indican que la FSH y el desarrollo folicular, no limitan el restablecimiento de la actividad reproductiva postparto. Se sabe que el amamantamiento inhibe la secreción de $\mathrm{GnRH}$ en hipotálamo porque incrementa los efectos negativos de los opioides endógenos y del estradiol en hipotálamo e hipófisis (Pérez et al., 2001). Desde un punto de vista fisiológico, este atraso en la 
ciclicidad ha sido asociado con la presencia de ciertos péptidos (endorfinas), los cuales inhiben la secreción de GnRH y en consecuencia, la liberación de LH. En la medida que aumenta el periodo vacío posparto el efecto bloqueador de estos péptidos disminuye y las vacas reinician su actividad reproductiva (González et al., 2006). La secreción tónica de LH por efecto del amamantamiento está en los centros neurales y es atribuido específicamente a sinapsis somato sensorial, originado a nivel de la teta y único para terneros amamantando. Se desconoce el mecanismo por el cual el amamantamiento inhibe la secreción pulsátil de $\mathrm{LH}$, pero se postula que la presencia de la cría es importante. Según Hoffman et al., (1996) estímulos sensoriales aún no bien determinados, inducidos por el amamantamiento o la presencia del ternero, atenúan la liberación hipotalámica de GnRH a la circulación portal o la capacidad de la hipófisis para responder apropiadamente al estímulo de la $\mathrm{GnRH}$. Estudios realizados por Griffith y Williams, (1996) con vacas normales ciegas y anósmicas que amamantaban sus propias crías o crías ajenas o que no amamantaban, confirman la disminución de la secreción de $\mathrm{LH}$ en vacas que amamantan, solamente cuando la madre reconoce su cría. Las vacas ciegas y anósmicas que amamantaron sus crías y las intactas que amamantaron crías ajenas presentaron aumentos de la frecuencia de pulsos de LH durante el tratamiento $(4.6+0.3$ pulsos en cinco horas) comparado con frecuencias bajas de los pulsos de LH $(1.2+0.01$ pulsos en cinco horas) en las vacas intactas que amamantaron sus crías. La eliminación de la visión y del olfato impidió a las vacas identificar sus crías y sufrir el efecto negativo del amamantamiento sobre la secreción de LH. En este sentido Myers et al., (1989) anularon el efecto del amamantamiento sobre la disminución de LH al aplicar un inhibidor de opioides endógenos (naloxona) y sugirieron que el amamantamiento, al menos parcialmente, inhibe la secreción de LH a través de un mecanismo que involucra a los opioides endógenos.

Ferreira, (1990) reporta un intervalo de parto al primer estro de 104 días para vacas amamantando contra 74 días para aquellas ordeñadas dos veces al día. Según Short et al., (1972) observaron el primer estro posparto en vacas Aberdeen Angus a los 65 días para vacas amamantando y 25 días para vacas cuyas crías 
fueron separadas a los dos meses. Las vacas tipo carne (Angus $x$ Hereford) que no amamantan ni tienen contacto con sus crías, pueden presentar su primera ovulación postparto más temprano (14.3 días posparto) que las que no amamantan, pero tienen contacto visual, táctil y olfativo con ellas (22.5 días) y las que amamantan libremente (35.4 días). Esto sugiere que la presencia de la cría, independientemente del amamantamiento prolonga el periodo anovulatorio (Hoffman et al., 1996). De manera similar, las vacas gemelas que se ordeñan y no tienen contacto con sus crías, presentan aumentos del nivel de LH más temprano y desarrollan un anestro postparto más corto que sus gemelas amamantando, indicando un efecto del amamantamiento y no de la lactancia sobre el nivel de LH y primera ovulación postparto (Smith et al., 1981).

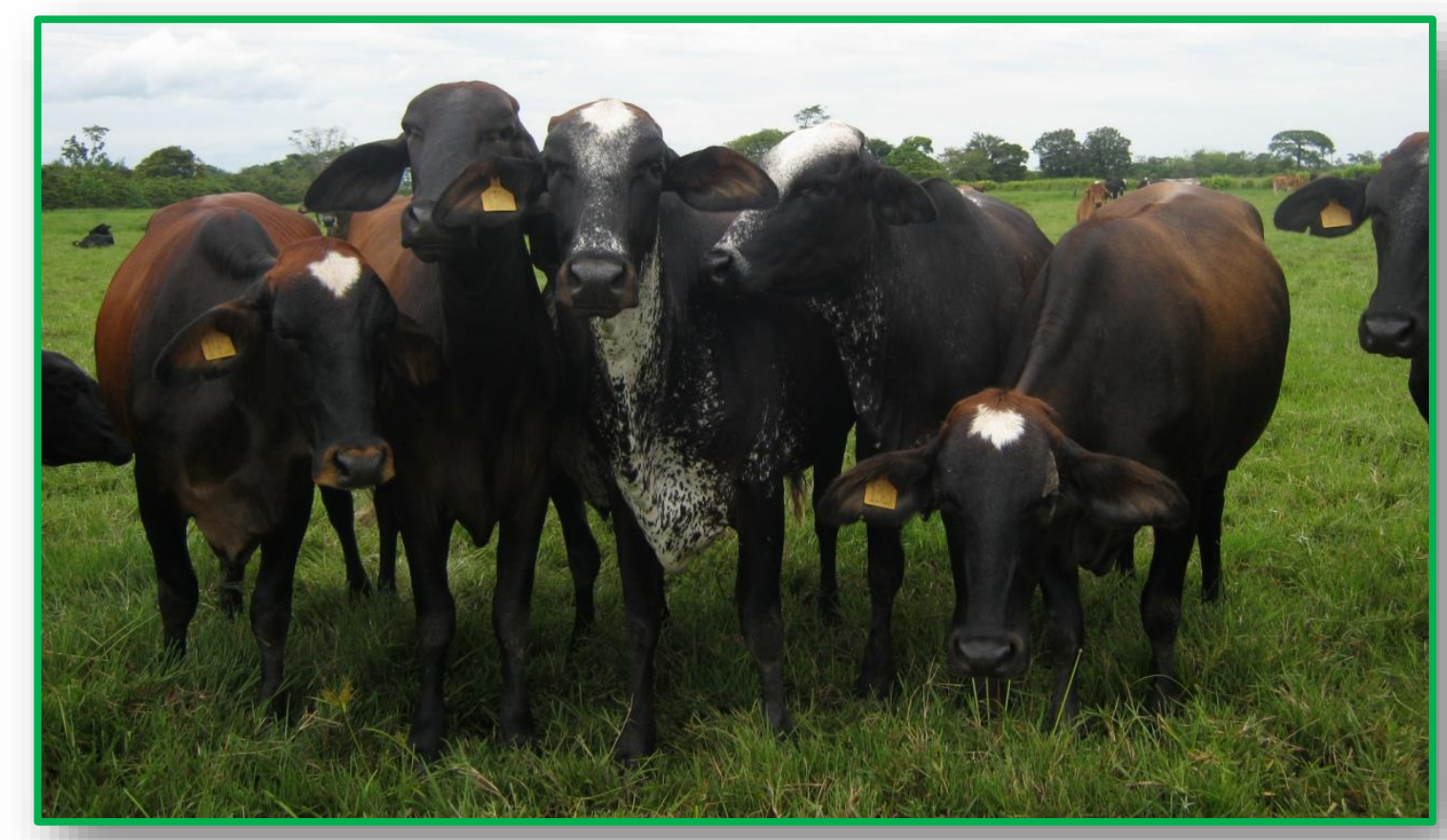

Fotografía 2. Las vacas con buena condición corporal al parto pueden perder determinado peso al inicio de la lactancia

Fotografía: María Ligia Roa Vega (2011)

En la mayoría de los mamíferos, después del parto, el estímulo del amamantamiento de la cría induce un período sin ciclos estrales, conocido como anestro postparto, cuya finalidad es permitir que la madre se recupere de los efectos de la preñez y que asegure la supervivencia de su cría (McNeilly, 1997). 
Durante el posparto, el eje hipotálamo-hipófisis-gónadas-útero debe recuperase en su total funcionamiento, para que se instale la nueva gestación. La extensión de este período con frecuencia resulta en pérdidas económicas para los productores y es responsable, en parte, de la baja eficiencia reproductiva de las vacas de doble propósito en el trópico (Villa y Arreguín, 1993; Williams et al., 1996).

Es suficientemente conocido que la interrupción de la lactancia provoca un doble efecto positivo sobre el comportamiento reproductivo posterior de los vientres. Por un lado, el menor drenaje de nutrientes se expresa en una rápida mejora de la condición corporal, alcanzándose altos índices de preñez y menores intervalos entre el parto y la concepción siguiente. Por otra parte, en el caso de los destetes definitivos o con apartes (adelantados, precoces o temporarios) se anulan los procesos inhibitorios, derivados de la presencia del ternero al pie, que afectan la reanudación temprana de los ciclos estrales en el posparto (Stahringer, 2006). Según Warren et al., (1988) las vacas con buena condición corporal al parto pueden perder determinado peso al inicio de la lactancia, por la movilización de reservas corporales, sin sacrificar la eficiencia reproductiva con manifestaciones tempranas de estro, independientemente del amamantamiento.

Una de las pocas ventajas fisiológicas en el inicio de la actividad reproductiva posparto es que se favorece el proceso de involución uterina, así como la reducción en la incidencia de infecciones uterinas. Sobre este particular, se puede señalar el efecto de la hormona oxitocina, liberada durante los episodios del amamantamiento, ya que podría contribuir en el proceso de involución uterina a través de la estimulación de las contracciones del músculo uterino. La respuesta contráctil del útero solamente puede darse en presencia de receptores específicos a la oxitocina. La formación de estos receptores se puede estimular por las bajas concentraciones circulantes de estradiol (estrógenos) provenientes de los pequeños folículos ováricos durante el periodo de acíclia. En forma contraria, las contracciones uterinas pueden ser deprimidas por otra hormona ovárica como la progesterona, liberada por el cuerpo lúteo después de una temprana ovulación posparto. Salgado et al., (2003) en un estudio realizado en doble propósito 
reportaron efecto del amamantamiento sobre la tasa de gestación hasta los cuatro meses posparto, encontrando valores de 70 y $20 \%$ para vacas con amamantamiento restringido, versus, tradicional, respectivamente. Igualmente, encontraron efecto del sistema de amamantamiento sobre los días abiertos, con valores de $109+52.93$ y $161.87+60.14$ para las vacas con amamantamiento restringido, versus, tradicional, respectivamente.

\section{SISTEMAS DE AMAMANTAMIENTO RESTRINGIDO}

Durante el análisis de los diferentes datos reproductivos en la ganadería doble propósito se estima que los días abiertos son de las principales circunstancias de pérdida económica para este sector, es por ello que se utilizan diferentes métodos de amamantamiento restringido con el fin de disminuir los días abiertos y poder conseguir ternero por año.

\section{Destete precoz}

La leche es al comienzo de la vida, el único alimento que consume el ternero y luego gradualmente va perdiendo importancia al aportar progresivamente menos a la dieta, por lo cual las ganancias de peso pasan a depender más de la calidad y cantidad de pasto que ingiere (Rovira, 1997). A mayor consumo de leche durante la etapa de cría, mayor será el peso del ternero al momento del destete convencional, demostrándose la habilidad materna. La práctica del destete precoz consiste en la separación de los terneros de las madres en forma anticipada al destete tradicional, la edad habitual de destete en este sistema doble propósito oscila entre los seis y ocho meses, con el objetivo de favorecer la recuperación del vientre productivo; permitiendo liberar a las hembras de los exigentes requerimientos de lactación y mejorar así la performance reproductiva. El ternero debe ser atendido buscando la manera de satisfacer sus requerimientos en ausencia de la leche materna. Para poder llevar adelante esta práctica con éxito se requiere un alimento que cubra todos los requerimientos del ternero en las etapas iníciales de su desarrollo. Ello permite que la vaca destine la energía de la producción de leche a otras funciones, como la actividad sexual. Se acepta como 
norma el hacerlo cuando el ternero tiene dos meses de edad y pesa no menos de $70 \mathrm{~kg}$ entre los 60 y 110 días de edad (Pérez et al., 2001).

Laster et al., (1973) destetaron precozmente los terneros 8 días antes del inicio de la temporada de servicio con edades que variaban entre 34 y 76 días. El destete incrementó la tasa de concepción en $26 \%$ en vaquillonas de primera parición Otros también lograron reducir la duración del anestro posparto en vacas pluríparas (Bellows et al., 1974; Houghton et al., 1990). Aun cuando fue implementado en condiciones climáticas rigurosas, el destete precoz mejoró significativamente la eficiencia reproductiva (Ray et al., 1973).

El destete precoz es más para la vaca que para el ternero. El inconveniente de este sistema es el manejo del ternero destetado precozmente, que requiere una suplementación lo que significa un incremento de costo para alimento balanceado y mano de obra (Monje, 2006). Asimismo, puede haber una reducción de la ganancia de peso del ternero, no alcanzando pesos comparables a los terneros que permanecieron al pie de la madre hasta la edad del destete tradicional (Arias et al., 1999).

\section{Destete temporal}

El destete temporal es otro sistema mediante el cual se ha intentado mejorar los índices reproductivos posparto. En esta modalidad de amamantamiento, el becerro a los 30,60 o 90 días de edad, se separa de la madre por períodos variables (48, 72 o 96 h). Esta separación del becerro de su madre, incrementa la secreción de LH e induce la ovulación y el estro (Williams et al., 1993). Los resultados obtenidos con este sistema han sido variables. Así por ejemplo, algunos autores encontraron que mientras un grupo de vacas posparto sometido a un destete temporario de 48 horas de duración presentaba un $44 \%$ de hembras preñadas 21 días después del tratamiento, el grupo testigo sólo alcanzó un 17\% de preñez (Smith et al., 1979). Otros observaron que, si bien el destete temporario por 48 horas no incrementaba los porcentajes de preñez al final de la temporada de servicio, lograba incrementar el número de vacas en celo a los 21 días 
posteriores al tratamiento (Tervit et al., 1982). Sin embargo, otros investigadores no lograron demostrar incrementos en los porcentajes de preñez utilizando este sistema (Makarechian y Arthur, 1990; Pace y Sullivan, 1980).

Diversos experimentos utilizando destete temporal se han sido realizados en la EEA INTA Balcarce (Alberio et al., 1984). En estos trabajos se logró mejorar los índices de preñez con la utilización de un destete temporal de 72 horas. Sin embargo, los resultados del destete temporal estaban afectados por diversos factores, como el intervalo parto-tratamiento, la condición corporal (primíparas vs pluríparas), por lo que el mismo no se ha encontrado muy extendido en las producciones cubanas, aunque pudiera hallarse con alguna frecuencia en algunos lugares de Europa así como en América Latina, sin constituirse esta técnica en la más aplicada en estas regiones.

La aplicación de la interrupción temporal del amamantamiento en fincas de la microrregión Sabanas de Bolívar, Córdoba y Sucre, permitió aumentar el número de vacas en calor y preñadas, obteniéndose en el sistema "doble propósito" 19 crías y 19 lactancias adicionales por cada 100 vacas sometidas a tratamiento y en el sistema "cría" 19 terneros adicionales por cada 100 vacas sometidas al tratamiento, lo que representó un aumento en el ingreso neto (Prieto et al., 2000).

La respuesta al destete temporal es muy variable, pues depende del tiempo posparto en que se realiza, de la condición corporal de la vaca y la duración del destete. Para eliminar de manera total, los efectos negativos del amamantamiento del becerro, es necesario separar completamente al becerro de la vaca por más de $96 \mathrm{~h}$, pues cuando el becerro se separa por $48 \circ 72 \mathrm{~h}$, al retornar el becerro con su madre los efectos negativos del amamantamiento se vuelven a presentar. (Shively y Williams, 1989)

\section{Amamantamiento una vez por día}

Otro sistema utilizado para reducir la duración del anestro posparto es el amamantamiento una vez por día por un período de 30 a 60 minutos. Randel, (1981) restringiendo el período de amamantamiento a 30 o 60 minutos por día 
(amamantamiento restringido) observó una disminución del intervalo parto-celo de 168 a 69 días en vaquillonas de primera parición con ternero al pie. Reeves y Gaskins, (1981) han descrito reducciones de la duración del anestro posparto de 20 días; sin embargo, observaron un incremento de la incidencia de celos cortos en los animales sometidos al amamantamiento una vez por día. Si bien estos autores observaron un efecto negativo sobre la ganancia de peso del ternero cuando se efectuaba un amamantamiento diario; Randel, (1981) no encontró un efecto de este amamantamiento restringido sobre la ganancia de peso del ternero. En trabajos posteriores Browning et al., (1994) mostraron que existe una menor ganancia de peso del ternero en la primera semana del amamantamiento una vez por día, pero aumentan a la segunda semana encontrando pesos al destete similares entre terneros que amamantaron una vez por día y los que lo hicieron ad libitum, por lo que esta técnica se avizora como una de las más prometedoras para su uso en el trópico, donde su uso se viene extendiendo desde hace algunos años.

\section{Enlatados "destetadores"}

Otro método para acortar el anestro posparto es la restricción del amamantamiento mediante la aplicación de placas nasales por un período variable, también conocido como tabla destetadoras. Trabajos llevados a cabo en Zimbabwe, mostraron que el enlatado de terneros por un período de ocho días redujo el intervalo posparto en vacas con alto nivel nutricional (Holness y Hopley, 1978) y el intervalo entre partos (Holness et al., 1980). Con un período de enlatado de 21 días mostraron incrementos en los índices de preñez, especialmente en establecimientos con índices reproductivos bajos (AACREA, 1991). En la Estación Experimental Agropecuaria INTA Colonia Benítez en Argentina se realizaron también trabajos para evaluar sistemas de manejo para disminuir el efecto negativo del amamantamiento del ternero sobre los índices reproductivos de la vaca de cría. Este estudio se realizó espacialmente con esta técnica del enlatado. La tecnología empleada por ellos consistió en una restricción temporaria del amamantamiento mediante la colocación en los ollares del ternero de una 
máscara plástica o metálica (lata) por períodos variables, que van desde los 7 hasta los 21 días. A través de estos trabajos se buscó evaluar los efectos del enlatado sobre algunos parámetros reproductivos de la vaca de cría, como así también sobre parámetros productivos del ternero. Los trabajos se llevaron a cabo con grupos de 40 a 50 vacas cruzadas con cebú primíparas (un período) y pluríparas (dos períodos). El enlatado se realizaba a partir de los 60 días de edad del ternero y una vez iniciada la temporada de servicio. Esto es muy importante, ya que a partir de esa edad, el ternero alcanza una maduración suficiente de sus compartimientos gástricos que le permite procesar alimentos fibrosos. Estos trabajos arrojaron resultados muy similares a los obtenidos en Zimbabwe y que se reflejan en la primera parte de esta referencia.

Como se evidencia esta tecnología pudiera convertirse en una posibilidad en un futuro, pero para Cuba y otros países del área, ésta se comporta aun como una posibilidad futura ya que como plantea Stahringer, (2006) para la implementación de esta tecnología con resultados satisfactorios, se requiere de un equipamiento médico veterinario y material significativo lo cual constituye una limitante importante a la hora de su establecimiento como principal medida para contrarrestar el anestro posparto en el ganado bovino de carne.

\section{Amamantamiento retrasado}

El amamantamiento retrasado consiste en realizar el amamantamiento del becerro ocho horas después del ordeño y surge de la necesidad de reducir los períodos prolongados de anestro postparto en vacas Bos taurus x Bos indicus manejadas en el sistema de doble propósito, sin afectar la producción de leche ni el desarrollo del becerro. Para lograrlo, las vacas se ordeñan de manera total en la mañana, previo apoyo del becerro. Después del ordeño, las vacas se van a pastorear a la pradera y los becerros permanecen en un corral con acceso a una pradera pequeña, donde consumen forraje y agua a libre acceso. A las ocho horas después del ordeño, las vacas y becerros se reúnen por 30-60 minutos, para que el becerro consuma toda la leche sintetizada en las ocho horas existentes entre el ordeño y el amamantamiento. Con este manejo de vacas y becerros, se simula 
realizar dos ordeños, pero la leche de la mañana es para el productor y la de la tarde para el becerro. Se recomienda iniciar el retraso del amamantamiento a partir de los siete días de edad de los becerros, para que éstos consuman calostro y leche a libre acceso en la primera semana de edad y disminuir la incidencia de enfermedades.

Con esta modalidad de amamantamiento realizado con vacas Bos taurus $\mathrm{x}$ Bos indicus manejadas en el sistema de doble propósito, se ha disminuido el intervalo parto primera ovulación de 77,1 + 20 días en vacas con amamantamiento restringido por dos horas al día, a 57,3 + 20 días (Pérez et al., 1991). Además, en este mismo estudio se encontró que el amamantamiento retrasado permitió que el $100 \%$ de las vacas presentaran su primera ovulación en los primeros 100 días postparto, comparado con $84 \%$ en las vacas con amamantamiento restringido. Resultados similares han sido obtenidos por Gallegos et al., (1990). Estos resultados apoyan la hipótesis de que las vacas de doble propósito que amamantan a su becerro después del ordeño, están sujetas a dos fuertes estímulos inhibitorios que se presentan uno después del otro. El primero, es causado por el proceso del ordeño (sujeción de la vaca, apoyo del becerro para el descenso de la leche y ordeño manual con el becerro al lado y el segundo, es provocado por el amamantamiento del becerro (por 30, 60 ó 120 minutos, después del ordeño). Al separar la presentación de estos dos estímulos, mediante el amamantamiento retrasado, se disminuye el intervalo parto primera ovulación y se incrementa la proporción de vacas que ovulan en los primeros 100 días posparto. El intervalo parto primera ovulación en vacas con amamantamiento retrasado se ha reducido aún más $(\mathrm{P}<0.05 ; 46.0$ + 12.1 días $)$, si además de retrasar el amamantamiento del becerro, las vacas se exponen al toro con pene desviado desde 7 días posparto (Pérez et al., 1993). La cantidad de leche ordeñada obtenida en vacas con amamantamiento retrasado, es cerca de $2 \mathrm{~kg}$ mayor que la de vacas con amamantamiento restringido $(7.04+0.5 \mathrm{~kg} /$ día vs $5.19+0.7 \mathrm{~kg} /$ día $)$, cuando se proporcionan 2 a 3 kg/vaca/día de complemento alimenticio con 17\% de proteína cruda (Gallegos et al., 1993). Por el contrario, la producción es similar, si las vacas reciben sólo $1 \mathrm{~kg} / \mathrm{vaca} /$ día de complemento alimenticio $(6.5+0.9$ 
kg/día vs $5.8+0.9$ kg/día; Pérez et al., 1993). La producción de leche total, obtenida al sumar la leche ordeñada y la consumida por el becerro, se incrementó en alrededor de $2 \mathrm{~kg} /$ día en vacas con amamantamiento retrasado con respecto a vacas con amamantamiento tradicional, cuando las vacas se suplementan con 1 $\mathrm{kg} /$ animal/día de concentrado comercial con $16 \%$ de proteína cruda al momento del ordeño y $2 \mathrm{~kg} / \mathrm{animal} / \mathrm{día}$ de complemento alimenticio, elaborado con melaza, gallinaza, sal común y sal mineral, al momento del amamantamiento $(11.3+1.6$ kg/día Vs $9.2+1.9$ kg/día) (Martínez et al., 1995).

La mayor producción de leche obtenida en vacas con amamantamiento retrasado con respecto a amamantamiento restringido, se debe a que el amamantamiento retrasado simula la realización de dos ordeños con presencia del becerro, lo cual debe incrementar la secreción de oxitocina. Esta sugerencia se apoya en: 1) cuando las vacas se exponen a un becerro ajeno, éstas secretan menos cantidad de oxitocina, con respecto a vacas expuestas con su propio becerro (Silveira et al., 1993); 2) al combinar el amamantamiento del becerro con el ordeño, a principios de la lactancia, se incrementa la producción de leche total (Bar et al., 1995); y 3) ordeñar las vacas por la mañana, con máquina y amamantar al becerro por la tarde, durante las primeras ocho semanas de la lactancia, aumenta la producción de leche total en $18 \%$, en lactancias de 300 días y dos ordeños diarios (Fulkerson et al., 1978).

El amamantamiento retrasado no disminuye la ganancia de peso de los becerros, sino que por el contrario, la incrementa (Gallegos et al., 1993). Cuando a los becerros se les proporciona alimento complementario a libre acceso, como se sugiere realizar en becerros de vacas con amamantamiento restringido, los becerros con amamantamiento retrasado ganan más peso $(827+34.8 \mathrm{~g} / \mathrm{días})$, con respecto a becerros de vacas con amamantamiento restringido $(702+26.2$ g/días) (Gallegos et al., 1991; 1993). Sin embargo, los resultados, de la mejora en la ganancia de peso de los becerros con amamantamiento retrasado son más evidentes cuando la alimentación de los becerros se basa en leche que consumen de la madre y forraje en la pradera, sin ningún complemento alimenticio (como la 
mayoría de los productores realiza en las explotaciones de doble propósito) (Orihuela et al., 1990). Al respecto Pérez et al., (1991; 1993) encontraron que los becerros con amamantamiento retrasado tuvieron mayor ganancia diaria de peso (779 + 862 g/día), comparado con becerros con amamantamiento restringido (576 + 608 g/día). A su vez, la ganancia de peso de becerros con amamantamiento retrasado $(724+100 \mathrm{~g} /$ día $)$ son similares a la de becerros que permanecen con sus madres durante 8 horas al día en la pradera (637 + 200 g/día) y por lo tanto, tienen la oportunidad de mamar a libre acceso (amamantamiento tradicional) (Martínez et al., 1995). Los resultados anteriores, en conjunto, indican que, a diferencia del amamantamiento restringido, en donde se disminuye el desarrollo del becerro, a menos de que se proporcione adecuada suplementación con alimento de buena calidad, el amamantamiento retrasado 8 horas después del ordeño no disminuye el crecimiento del becerro, sino que por el contrario la mejora (Gallegos et al., 1990 y 1993; Pérez et al., 1991; 1993) y permite obtener pesos de $114-124 \mathrm{~kg}$ a los 100 días de edad. Los becerros con amamantamiento retrasado maman menos tiempo (19.5-32 minutos) y en menor frecuencia (1.1-3.2 veces), con respecto a becerros con amamantamiento restringido (22.9-39 min y 3.3-3.8 veces) (Gallegos et al., 1991). Esta menor frecuencia e intensidad de amamantamiento de los becerros a las vacas con amamantamiento retrasado explican, en parte, su más pronto restablecimiento de la actividad ovárica posparto y cuestionan la necesidad de dejar al becerro junto con su madre por períodos mayores de 30 minutos después del ordeño.

\section{CONCLUSIONES}

Los efectos negativos del amamantamiento sobre la función reproductiva posparto, se producen principalmente en el hipotálamo, donde disminuyen la secreción de $\mathrm{GnRH}$ y LH en hipófisis. Existen claras evidencias indicando que la FSH y el desarrollo folicular en el ovario, no limitan el restablecimiento de la actividad reproductiva postparto. La presencia constante del becerro sin mamar incrementa la secreción de cortisol y oxitocina; pero no se conoce la participación 
de estos cambios endocrinos en el restablecimiento de la actividad reproductiva de las vacas postparto.

El amamantamiento continuo o la interacción constante de la vaca con su becerro prolongan el período de anestro posparto. La sola presencia del becerro sin mamar inhibe el restablecimiento de la actividad reproductiva y prolonga el anestro postparto; por lo cual, las estrategias de manejo para disminuir el anestro posparto, deben enfocarse a manipular el amamantamiento del becerro y separar, lo más posible, al becerro de la vaca y romper el efecto de la asociación vacabecerro.

El destete precoz elimina de manera total el estímulo del amamantamiento y reduce el período de anestro postparto; sin embargo, su implementación es difícil para los ganaderos del sistema productivo doble propósito, a menos que dispongan de mayor nivel tecnológico y de vacas que no requieran del estímulo del becerro para la eyección de la leche, actualmente en la región Orinoquia los cruces Bos indicus x Bos taurus repercuten en el efecto sensorial para el descenso de la leche. Adicionalmente, destetar el becerro a los pocos días de edad, disminuye la producción de leche, reduce la duración de la lactancia y el desarrollo corporal del becerro.

El amamantamiento retrasado es una opción viable que el productor de ganado bovino de doble propósito puede implementar para disminuir el anestro posparto, sin afectar negativamente la producción de leche y los cambios de peso corporal de las vacas. Además, el amamantamiento retrasado permite un desarrollo adecuado de los becerros, sin necesidad de alimentación extra.

Para implementar alguna de las estrategias de manejo del amamantamiento y mejorar la eficacia productiva y reproductiva de las explotaciones bovinas de doble propósito en trópico, se debe considerar la no disminución del desarrollo del becerro y el mantenimiento o incremento de la duración de la lactación y la producción de leche por parte de la vaca. 
Es necesario realizar evaluaciones de tipo económico, directamente con los productores, para determinar las opciones de manejo del amamantamiento que permitan aumentar los ingresos económicos, mediante una mejor eficacia productiva y reproductiva de las explotaciones. También se requiere determinar si mediante una adecuada suplementación de vacas y becerros se puede disminuir aún más la duración del anestro postparto y mejorar la producción de leche y el desarrollo del becerro de las vacas con amamantamiento retrasado.

\section{REFERENCIAS BIBLIOGRÁFICAS}

1. AACREA. Uso de "enlatado temporario" de terneros durante la época de servicio. Resumen de experiencias. Manejo de campo natural y otros temas. Vol. 1:36-38. XII Reunión Zonal. AACREA Regional. 1991.

2. Acosta G., Tarnavsky K., Platt E., Hamernik L., Brown L., Schoeneman, Reeves J. Nursing enhances the negative effect of estrogen on th release in the cow. J ANIM. SCI. 57: 1.530-1.536. 1983.

3. Albeiro H., Butler M., Palma G., Mihura H., Torquati O. Efecto de un destete temporario sobre la reactivación sexual posparto de vacas de cría multíparas. Rev. Arg. Prod. Anim. 1984.

4. Arias M., Soni A., Stahringer C., Sampedro D., Slobodzian A. Optimizando la eficiencia biológica en la reproducción. Jornada Ganadera del NEA. 1999.

5. Bar P. U., Maltz E., Bruckental I., Folman Y., Kali Y., Garcitua H., Lehrer R., Knight H., Robinzon B., Voet H., Tagari H. Relationship between frequent milking or suckling in early lactation and milk production of high production dairy cows. J. Dairy Sci. 1995.

6. Bellows A., Short E., Urick J., Pahnish F. Effects of early weaning on postpartum reproduction of the dam and growth of calves born as multiples or singles. J. Anim. Sci. 39. 1974.

7. Berardinelli G., Joshi S. Initiation of postpartum luteal function in primiparous restricted-suckled beef cows exposed to a bull or excretory products of bulls or cows. American Society of Animal Science. 2005.

8. Bertha P., Mireya V. Fisiología de la reproducción: Hormona liberador de gonadotrofinas. Rev Fac Med UNAM. Vol. 45. 2002.

9. Browing R., Robert B. S., Lewis A. W., Randel R. D. Effects of postpartum nutrition and once-daily suckling on reproductive efficiency and preweaning calf performance in fall-calving Brahman (Bos indicus) cows. J Anim Sci. 1994.

10. Cavestany D. Fisiología del puerperio. Serie de reproducción animal, tema 1: post-parto en la hembra bovina. Publicación Miscelánea (IICA). No. 644. Montevideo (Uruguay). 1985.

11. Corbellini C., Busso F. La Vaca lechera antes y después del parto. $1^{\text {a }}$ ed. Buenos Aires, Ediciones INTA. 2011.

12. Corbellini C., Busso V. La vaca lechera antes y después del parto: puntos a seguir para lograr una transición efectiva. Edición Rambeaud. $1^{\text {a }} \mathrm{ed}$. Ediciones INTA: Buenos Aires. 2011. 
13. Domínguez C., Ruiz Z., Pérez R., Martínez R., Drescher K., Pinto L., Araneda R. Efecto de la condición corporal al parto y el nivel de la alimentación sobre la involución uterina, actividad ovárica, preñez y la expresión hipotalámica y ovárica de los receptores de leptina en vacas doble propósito. Universidad experimental Rómulo Gallegoz. 2008.

14. Ferreira M. Efeit da amament e çäo e do nivel nutricional na atividade ovaria na de vacas mestiças leiteras. Viçosa. Dissertaçäo (doutorado em Zootecnia) Universidade Federal de Viçosa. 1990.

15. Fulkerson W., Hooley R., Findlay J. Improvement in milk production of first calf heifers by multiple suckling. Australian Journal of Agricultural Research. 1978.

16. Galeano R. Evaluación genética del recurso animal de los sistemas de producción de bovinos en doble propósito en Colombia. Tesis Maestría, Universidad Nacional de Colombia. 2010.

17. Gallegos J., Pérez H., Solaris M., García M., Osorio M. Comportamiento productivo y reproductivo de vacas de doble propósito en dos sistemas de amamantamiento en el trópico. Instituto de recursos genéticos y productividad, Colegio de posgraduados. México. 1991

18. Garcia M., Imakawa J., Day L., Zalesky D., Kittock J., Kinder E. Effect of suckling and ovariectomy on the control of luteinizing hormone secretion during the pospartum period in beef cows. Biol. Reprod. 31, 771-778. 1984.

19. Gaona C., Rómulo H., Erika A. Relación nutrición fertilidad en Bovinos: un enfoque bioquímico y fisiológico. Palmira: Universidad Nacional de Colombia. 2008.

20. Gareth W., Xue J., Joanne C., Joanne A. Harrold. Anabolic neuropeptides, Diabetes and Endocrinology Research Group, Department of Medicine, University of Liverpool, Duncan Building, Daulby Street, Liverpool. Physiology \& Behavior 81. 2004.

21. Gigli I., Russo A., Agüero A. Consideraciones sobre la dinámica ovárica en equino, bovino y camélidos sudamericanos. InVet v.8 Facultad de Ciencias Veterinarias, Buenos Aires. 2006.

22. GOBERNACIÓN DEL META, SECRETARÍA TÉCNICA REGIONAL. Cadenas productivas del Meta. Villavicencio. 2008.

23. GOBERNACIÓN DEL META, SECRETARIA DE AGRICULTURA. Evaluaciones Agropecuarias, Secretaría de Agricultura y Ganadería. 2007.

24. Gómez D. F. Destete precoz, vigor y salud del ternero. Cartilla INIA, Uruguay. 2008

25. González C., Goicochea J., Rodríguez A., Madrid N., González D. Incorporación al servicio en novillas mestizas doble propósito. Arch. Latinoam. Prod. Animal. 14 (1): 1-9. 2006.

26. Griffith K., Williams L. Roles of maternal vision and olfaction in suckling- mediated inhibition of LH secretion, expression of maternal selectivity, and lactational performance of beef cows. Biol. Reprod. 54, 761-768. 1996.

27. Hafez, E. Reproducción e inseminación artificial en animales. 6 ${ }^{\underline{a}}$ ed., Interamericana McGraw-Hill, México. 1996.

28. Hafez B. Reproduction in Farm Animals. Seventh edition. Donna Balado USA. 2000.

29. Henao G., Trujillo L., Maldonado G. Liberación de gonadotropinas hipofisiarias y factores que la afectan durante el posparto bovino. Rev Col Cien Pec., 13 (1). 2000 
30. Hinshewood M., Dierschke D., Hauser E. Effect of suckling on the hypothalamic pituitary axis in postpartum beef cows, independent of ovarian secretions. Biol. Reprod. 32: 290-300. 1985.

31. Hoffman D. P., Stevenson J. S., Minton J. E. Restring calf presence without suckling compared with weaning prolongs postpartum anovulation in beef cattle. J Anim Sci. 1996.

32. Holness $H$., Hopley $H$. The effects of plane of nutrition, live weight, temporary weaning and breed on the occurrence of oestrus in beef cows during the postpartum period. An i t a. Prod. 26: 4 7. 1978.

33. Holness $H_{\text {. }}$, Hale $H$., Hopley $H$. Ovarian activity and conception during post partum period in Afrikander and Mashona cows. Zimbabwe J. Agric. 1980.

34. Houghton L., Lemennager P., Horstman A., Hendrix S., Moss E. Effects of body composition, pre - and postpartum energy level and early weaning on reproductive performance of beef cows and preweaning calf gain. J. Anim. Sci. 68. 1990.

35. Izaguirre F., Martínez J. J., Sá O. L., Ramón C., Hernández P. H., Priego M. G. Influencia del amamantamiento y presencia del toro en el comportamiento productivo y reproductivo de vacas pardo suizo en el trópico húmedo. Rev. Cient. (Maracaibo). 17 (6). 2007.

36. Knowles T., Edwars D. A comparison of the effects of restricted suckling and artificial calf rearing systems on dam and calf performance. Malays. Agric. J. 54: 1-9. 1983.

37. Landaeta H. Etología y producción animal. Universidad del Zulia, Facultad de Ciencias Veterinarias, Departamento de Producción e Industria Animal. Unidad de Investigaciones Zootécnicas. Zulia. Mundo Pecuario, VII (3): 116-129. 2011.

38. Lascano C. E, Holmann, F. Conceptos y metodología de investigación en fincas con sistema de producción animal de doble propósito. Publicación CIAT No. 296. Cali: Consorcio Tropileche, 55. 1997.

39. Laster B., Glimp A., Gregory E. Effects of early weaning on postpartum reproduction of cows. J. Anim. Sci. 36. 1973.

40. Lidfors M., Jensen P., Algers B. Suckling in free-ranging beef cattle - temporal patterning of suckling bouts and effects of age and sex. Ethology. 1994

41. Little A., Anderson M., Durkin W. Influence of partial suckling of crossbred dairy cows on milk offtake and calf growth in the Ethiopian Highlands. Trop. Anim. Health Prod. 23: 108-114. 1991.

42. Makarechian P., Arthur F. Effects of body condition and temporary calf removal on reproductive performance of range cows. Theriogenology. 1990.

43. Malven V., Parfet R., Gregg W. Relationsships among concentrations of four neuropeptides and luteinizing hormone-releasing hormone in neural tissudes of beef cows following early weaning. Journal of Animal Science. 1984.

44. Margerision K., Preston R., Phillips C. Restricted suckling of tropic dairy cows by their own calf or other cows' calves. School of Agricultural and Forest Sciences, University of Wales, Bangor, Gwynedd LL57 2UW, United Kingdom; I center Inter-institutional for the Agricultural Development in the Valley of Cauca, Cali, Colombia; and Department of Clinical Veterinary Medicine, University of Cambridge, Cambridge CB3 0ES, United Kingdom. American Society Animal of Science. 2002.

45. McNeilly S. Lactation and fertility. J. Mammary Gland Biol. Neoplasia 2. 1997. 
46. Monje A. Destete precoz en cría vacuna. Recuperado en junio del 2006, Disponible

En: www.inta.gov.ar/concepcion/info/documentos/ganaderia/destete1.htm

47. Montaño E., Ruíz Z. ¿Por qué no ovulan los primeros folículos dominantes de las vacas cebú posparto en el trópico colombiano? Facultad Ciencias Agrarias, Universidad de Antioquia. 18 (2): 127-138. 2005.

48. Myers R., Myers A., Gregg W., Moss E. Endogenous opioid supression on release of luteinizing hormone during the suckling and postpartum anestrus beef cows. Dom Anim Endocrinol, 6: 183-190. 1989.

49. Orihuela A. Effect of calf stimulus on the milk yield of zebu-type cattle. Appl. Anim. Behav. Sci. 1990.

50. Pace M., Sullivan J. Effect of Synchro-Mate-B treatment (SMB) and calf separation on beef cattle estrus and pregnancy rates. J. Anim. Sci, 51. 1980.

51. Perea G. F., Cruz R., Gonzales R., Soto B. E., Gonzales E., Rincón E. Evaluación ultrasonográfica de la involución del cuello uterino en vacas mestizas tropicales. Universidad de Zulia. Facultad de Ciencias Veterinarias, Producción Animal. 1997.

52. Pérez P., Becerril M., Lamothe Z., Glafiro H., López S., Gallegos J. Efecto del amamantamiento retrasado en la actividad posparto de las vacas y en los becerros de doble propósito. Interciencia, 31 (10). 2006.

53. Pérez H., Sánchez del R. J., Gallegos S, Anestro postparto y alternativas de manejo del amamantamiento en vacas de doble propósito en trópico. Universidad Autónoma de México Colegio de Postgraduados, Especialidad de Ganadería, Departamento de Zootecnia, Universidad Autónoma Chapingo, (México) Invest. Agr.: Prod. Sanid. Anim. 16 (2). 2001.

54. Petryna A., Bavera G. A. Etología Cursos de Producción Bovina de Carne, FAV UNRC. 2002.

55. Preston R., Vacarro L. Dual purpose cattle production systems. In: New techniques in cattle production. Butterworths: Phillips J, 20-32. 1989.

56. Ptazynaska M. La importancia del periodo posparto y su impacto en la reproducción. 2007. Disponible En: www.cigal.biz.

57. Randel D. Effect of once-daily suckling on postpartum interval and cow-calf performance of first-calf Brahman X Hereford heifers. J. Anim. Sci. 1981.

58. Randel D. Nutrition and postpartum rebreeding in cattle. J. Anim. Sci. 1990.

59. Ray E., Roubicek B., Lane A., Hansen J., Theurer B., McGinty D. Suplementation or early weaning with beef heifers. Proc. West. Sect. Am. Soc. Anim. Sci. 1973.

60. Reeves J., Gaskiins T. Effect of once-a-day nursing on rebreeding efficiency of beef cows. J. Anim. Sci. 1981.

61. Román H. Situación actual y retos de la ganadería bovina en el trópico. XX Simposium de Ganadería Tropical: Alternativas de alimentación del ganado bovino en el trópico. México. 1995.

62. Rovira J. Manejo nutritivo de los rodeos de cría en pastoreo; destete. Montevideo, Uruguay: Hemisferio Sur. 1997.

63. Ruiz C. T., Olivera Á. M. Ovarian follicular dynamics in suckled zebu (Bos indicus) cows monitored by real time ultrasonography. Anim. Reprod. Sci. 54. 1999.

64. Salgado O., Simanca J., Gómez C. Amamantamiento restringido y suplementación sobre la eficiencia reproductiva en vacas del sistema doble propósito. Revista MVZ Córdoba, 12. 2007. 
65. Salgado O., Roger, Torregroza S., Lino Alverez P., Martínez H., Rugeles P., Vergara G., Maza A. Amamantamiento restringido y suplementación sobre los perfiles metabólicos en vacas del sistema doble propósito. Revista MVZ. Universidad de Córdoba. Facultad de Medicina Veterinaria y Zootecnia, Departamento de Ciencias Pecuarias. 2006

66. Salgado R., Álvarez J., Bertel M., Gonzáles M, Maza L., Torregoza L., Efecto de la época del parto y del sistema de amamantamiento sobre la eficiencia reproductiva de vacas del sistema doble propósito. Universidad de Córdoba, Facultad de Medicina Veterinaria y Zootecnia. Departamentos de Medicina Animal y de Zootecnia. Universidad de Sucre, Montería, Colombia. 2003.

67. Sandoval E., Valle A., Flores R., Medina R. Crecimiento ponderal en becerros de doble propósito sometidos a un sistema integral de crianza. Estación Experimental Falcón. Coro. Estado Falcón. CENIAP. Departamento de Estadística e Informática. Maracay. Estado Aragua. 1992.

68. Santos R., Arreguín A., Villa G. A., Román H. Interacción de la condición corporal y la frecuencia del amamantamiento sobre la actividad ovárica postparto en vacas cebú. El desarrollo folicular ovárico. Reunión Nacional de Investigación Pecuaria. México. 1995.

69. Sergio G. Resolución del anestro en el ganado bovino de carne. Programa de Reproducción Animal del C.E. Aldama-INIFAP-SAGAR. 2006.

70. Shively E., Williams L. Patterns of tonic luteinizing hormone release and ovulation frequency in suckled anestrous beef cows following varying intervals of temporary weaning. Domest. Anim. Endocrinol. 1989.

71. Short E., Bellows A., Moody L., Howland E. Effects of suckling and mastectomy on bovine postpartum reproduction. J. Anim. Sci. 34 (70). 1972

72. Short E., Bellows A., Staigmiller B., Berardinelli G., Custer E. Physiological mechanisms controlling anestrus and infertility in postpartum beef cattle. J Anim Sci. 1990.

73. Silveira A., Spoon A., Ryan P., Williams L. Evidence for maternal behavior as a requisite link in suckling-mediated anovulation in cows. Biol. Reprod. 1993.

74. Smith F., Burrell C., Shipp D., Sprott R., Songster N., Wiltbank N. Hormone treatments and use of calf removal in postpartum beef cows. J. Anim. Sci. 1979.

75. Stagg K., Diskin G., Sreenan M., Roche F. Follicular development in longterm anoestrous suckler beef cows fed two levels of energy postpartum. Anim. Reprod. Sci. 38: 49-61. 1995

76. Stahringer R. Anestro posparto y pubertad en bovinos de cría. 2006. Recuperado en junio del $2011 . \quad$ Disponible En: http://www.paraguayganadero.com/articulo.php?ID=158

77. Sumano H., Ocampo L. Farmacología clínica de los Bovinos. México, D. F.: Trillas. 1996

78. Tegegne P., Osuji O., Lahlou K. A., Mukasi M. E. Effect of dam nutrition and suckling on lactation in Borana cows and growth in their Borana $\times$ Friesian crossbred calves in an early weaning system in Ethiopia. Anim. Prod., 58: 19-21. 1994.

79. Tennant B., Kendendrick, Peddicord G. Uterine involution and ovarian function in the postpartum cow. A retrospective analysis of 2,338 genital organ examinations. Cornell Vet. 57: 543. 1967.

80. Tervit R., Smith F., Kaltenbach C. Postpartum anestrus in beef cattle: a review. Proc. N. Zeal. Soc. Anim. 1977. 
81. Van Engelen, G. M., Breeveld V., Everts M., Van Der Weyden, T. M., Rutten V. Cervical ripening and parturition in cows are driven by a cascade of proinflammatory cytokines. Reproduction in Domestic Animals, 44: 834-841. 2009.

82. Villa A., Arreguín A. Tecnología disponible y principales líneas de investigación para resolver el anestro posparto en vacas de doble propósito. XVI Simposium de Ganadería Tropical: 4ํ Ciclo de conferencias sobre bovinos de doble propósito. INIFAP. Veracruz, México. 1993.

83. Villagomez G., Arreguin A. Tecnología disponible y principales líneas de investigación para resolver el anestro postparto en vacas de doble propósito. En: XVI Simposium de ganadería tropical. 4ํㅜ Ciclo de conferencias sobre bovinos de doble propósito. INIFAP. Veracruz, México. pp. 55-84. 1993.

84. Villagómez E., Zárate M., Arellano M., Villa A., González E. Efectos de la suplementación energética y del amamantamiento sobre el desarrollo folicular y el anestro de vacas de doble propósito. XXXV Reunión Nacional de Investigación Pecuaria. Yucatán, México. 1999.

85. Warren C., Spitser C., Burns L. Beef cow reproduction as affected by postpartum nutrition and temporary calf removal. Department of Animal Science Clemson University Clemson, SC 29634-0361, USA. 1988.

86. Wehreden A. Centimetry and ultrasonographic observation of the cervix regression in Dairy cows Turing the first 10 days post partum. J Vet.Med A Physiol Pathol Cin Med. 2003.

87. Werth A., Whittier C., Azzam M., Deutscher H., Kinder E. Relationship between circulating progesterone and conception at the firts postpartum estrus in young primiparous beef cows. J. Anim. Sci. 74: 616-619. 1996.

88. Williams G. L. Suckiln as regulator of postpartum rebreeding catlee. J Anim Sci. 1990.

89. Williams L., McVey R., Hunter F. Mammary somatosensory pathways are not required for suckling-mediated inhibition of luteinizing hormone secretion and delayed of ovulation in cows. Biol. 1993.

90. Williams L., Gazal S., Guzmán V. A. y Stanko L. Mechanism regulating sucklingmediated annovulation in the cow. Anim. Reprod. Sci. 1996.

91. Yavas Y., Johnson H. y Walton S. Modification of follicular dynamics by exogenous FSH and progesterone, and the induction of ovulation using hCG in postpartum beef cows. Theriogenology 52: 949-963. 1999.

92. Yavas Y., Walton S. Postpartum acyclity in suckled beef cows: a review. Theriogenology 54: 25-55. 2000. 